\title{
New Implementation of a GMSK Demodulator in Linear Software Radio Receiver
}

\author{
Yik-Chung Wu and Tung-Sang $\mathrm{Ng}$ \\ \{ycwu, tsng\}@eee.hku.hk \\ Department of Electrical and Electronic Engineering \\ The University of Hong Kong \\ Pokfulam Road, Hong Kong \\ Tel.: $++852+28578406 \quad$ Fax: $++852+25598738$
}

\begin{abstract}
This paper proposes a practical linear software-radio architecture (dealing with linear modulations) that is suitable for multi-mode operation. In particular, it is shown how to integrate GMSK into the proposed architecture. Coherent and noncoherent detections of GMSK signals are detailed for the implementation of the proposed software radio.
\end{abstract}

\section{INTRODUCTION}

Multi-mode transceivers are very useful to achieve global roaming as different communication standards are used among different countries and territories. Software-radio implementation is a very promising technology for realizing multi-mode transceivers. In this paper, we propose a linear software-radio architecture that is suitable for realizing multi-mode terminals. By a linear software-radio architecture, it is meant that the software radio is targeted for dealing with linearlymodulated signals, such as BPSK, QPSK, OQPSK and MSK signals. This software radio is hence not complex to implement. The particular merit of the proposed linear software-radio architecture is that it can be extended to handle GMSK, which is a nonlinear modulation. The proposed software radio enables coherent and noncoherent detections of GMSK signals.

Section II describes the proposed linear softwareradio architecture. Application of this linear architecture for GMSK demodulation is detailed in Section III. Finally, conclusions are drawn in section IV.

\section{LINEAR SOFTWARE-RADIO RECEIVER ARCHITECTURE}

Software radio has its origin in military applications and one of the early survey papers is by Mitola [1]. Ideally, software radio is best operated by having the signal sampled at the $\mathrm{RF}$ and all demodulation and detection functions implemented in a generalpurpose processor. However, this software radio is not economically practical, at least at this moment. Practical software radios are usually implemented with IF sampling and with subsequent processing being performed in a combination of ASICs, FPGAs, DSPs and other microprocessors. Software radio architecture is an open one and different people have slightly different definitions. However, a practical one, as shown in Figure 1, usually consists of an A/D converter, a digital downconverter, a set of filters, an interpolator, a carrier recovery unit and a simple decision unit [2], [3], [4].

After the desired signal is frequency-shifted to the IF by using an analog mixer and a local oscillator, it is sampled using bandpass sampling, which performs down conversion as well. A replica of the signal is then produced near the zero frequency. Numerically contolled oscillator (NCO), which needs not be phase-coherent with the incoming carrier, shifts (down converts) the signal of the desired frequency band to the baseband (i.e., to perform channel selection). The baseband signal is then processed by a cascaded-integration-and-comb (CIC) filter and a multistage half-band filter (HBF), both of which remove the high frequency channels and perform decimation on the resultant signal. The effect of both filters is to suppress signals from other undesired channels without degrading the desired signal. This is achievable by designing the filters such that the passband of the combined filter is several times wider than that of the data rate. The effect of CIC filter and HBF can also be compensated at the digital matched filter for better performance. The decimated baseband signal is then filtered by a digital matched filter for waveform matching. Since the symbol timing of the incoming signal is asynchronous to the timing of the sampling clock, there is almost always a difference between their timings. An interpolator is therefore required to produce the samples at correct sampling instants in order to avoid intersymbol interference. 
Either carrier recovery or differential detection unit follows. They are put in the same block in Figure 1 as they both perform the same function: to remove the residual carrier-phase offset. Simple logic is used to recover the transmitted data.

It is easy to observe that linear modulations (BPSK, QPSK, OQPSK, etc.) can be accomodated into the architecture shown in Figure 1 with root-raisedcosine matched filter for maximing the SNR. It is also clear that simple threshold decision logic is used for making decision. For $\pi / 4-\mathrm{DQPSK}$, its $\pi / 4$ rotation and differential encoding make it a little bit more complicated than QPSK in demodulation. However, $\pi / 4-D Q P S K$ can also be adapted to the proposed software-radio architecture with rootraised-cosine matched filter, except that extra logic circuitry is required in order to deal with the $\pi / 4$ rotation and differential encoding. For MSK, it is well known that it can be expressed in the form of OQPSK using half-cycle sinusoidal pulses. The proposed architecture can therefore be applied to MSK signals with half-cycle sinusoidal pulse matched filter and with decision logic the same as that for OQPSK. We are going to discuss how to fit GMSK (a nonlinear modulation) into this architecture.

\section{GMSK DEMODULATION}

\section{A. GMSK and Linearized GMSK}

For continuous phase modulation (CPM), the signal is given by [5]

$$
s(t)=\sqrt{\frac{2 E}{T}} \cos \left(2 \pi f_{o} t+\phi(t, a)\right)
$$

where

$$
\phi(t, a)=2 \pi h \sum_{i=-\infty}^{\infty} a_{i} q(t-i T), \quad a_{i}= \pm 1 .
$$

In this expression, $h$ is the modulation index, $q(t)$ is given by $q(t)=\int_{-\infty}^{t} g(\tau) d \tau$ and $g(t)$ is a pulse over a finite interval $0 \leq t \leq L T$ satisfying $\int_{-\infty}^{\infty} g(\tau) d \tau=$ $1 / 2$. GMSK is a special case of CPM, in which $h=0.5$ and $g(t)$ is given by the convolution between a Gaussian pulse and a rectangular pulse, that is,

$$
\begin{aligned}
g(t) & =\frac{1}{2 T} \operatorname{rect}(t / 7) \otimes h_{G a u s s}(t) \\
& =\frac{1}{2 T}\left[Q\left(2 \pi B \frac{t-T / 2}{\sqrt{\ln 2}}\right)-Q\left(2 \pi B \frac{t+T / 2}{\sqrt{\ln 2}}\right)\right]
\end{aligned}
$$

where $Q(t)=\int_{t}^{\infty}(2 \pi)^{-1 / 2} e^{-\tau^{2} / 2} d \tau$, and $B$ is the $3 \mathrm{~dB}$ bandwidth of the Gaussian filter.
If a GMSK signal can be expressed in the form of OQPSK like that for a MSK signal, a linear demodulator can be used. However, it is shown in [6] that if $L \neq 1$, there is no solution for this problem. For practical implementation, however, approximation can be applied to GMSK signals. Laurent [7] has shown that any constant-amplitude binary phase modulation can be expressed as the sum of a finite number of time-limited amplitude modulated pulses. The fundamental result for CPM baseband decomposition, $s_{b}(t)$, is formulated as [7]

$$
s_{b}(t)=\sum_{N=-\infty}^{\infty} \sum_{K=0}^{2^{L-1}-1} \exp \left[j \pi h A_{K, N}\right] C_{K}(t-N T)
$$

where

$$
A_{K, N}=\sum_{n=-\infty}^{N} a_{n}-\sum_{i=1}^{L-1} a_{N-i} \alpha_{K, i}
$$

and

$$
C_{K}(t)=S_{0}(t) \times \prod_{i=1}^{L-1} S_{i+L \cdot \alpha_{K, i}}(t) .
$$

In the above expression,

$$
\begin{gathered}
K=\sum_{i=1}^{L-1} 2^{i-1} \alpha_{K, i}, \\
S_{n}(t)=\frac{\sin [\Psi(t+n T)]}{\sin (h \pi)},
\end{gathered}
$$

and $\Psi(t)= \begin{cases}h \pi \int_{-\infty}^{t} g(\tau) d \tau & t<L T \\ h \pi-h \pi \int_{-\infty}^{t-L T} g(\tau) d \tau & t \geq L T .\end{cases}$

For 0.3 GMSK, it can be easily shown that the response of a rectangular pulse upon a Gaussian filter, as indicated in (2), spans only 4 symbol periods (i.e. $\mathrm{L}=4$ ). It follows that

$$
\begin{aligned}
s_{b}(t) & =\sum_{N=-\infty}^{\infty} \exp \left[j \pi h \sum_{n=-\infty}^{N} a_{n}\right] C_{0}(t-N T) \\
& +\sum_{N=-\infty}^{\infty} \sum_{K=1}^{7} \exp \left[j \pi h A_{K, N}\right] C_{K}(t-N T)
\end{aligned}
$$

The 0.3 GMSK is composed of the superposition of eight impulses, that is, $C_{K}(t)$ for $0 \leq K \leq 7$. Numerical computation indicates that the main pulse $\mathrm{C}_{0}(\mathrm{t})$ for 0.3 GMSK contains $99.83 \%$ of the energy and for 0.5 GMSK, the main pulse even contains $99.98 \%$ of the total energy. Therefore, $C_{0}(t)$ is the most significant component and others can be neglected. Hence, it is reasonable to approximate a 0.3 GMSK signal with only the first term of (4). It follows that, when there is no data for $t<0$, we have 


$$
\begin{aligned}
s_{b}(t) & \approx \sum_{N=0}^{\infty} \exp \left[j \pi h \sum_{n=0}^{N} a_{n}\right] C_{0}(t-N T) \\
& =\sum_{k=0}^{\infty} b_{2 k+1} C_{0}(t-2 k T-T) \\
& +j \sum_{k=0}^{\infty} b_{2 k} C_{0}(t-2 k T)
\end{aligned}
$$

where $\quad b_{2 k}=b_{2 k-1} a_{2 k}, b_{2 k+1}=-b_{2 k} a_{2 k+1} \quad$ and $b_{-1}=1$.

The GMSK signal can be demodulated by a variety of methods, which can be grouped into two main categories: non-coherent detection (differential demodulation, either involving 1-bit or 2-bits, and limiter-discriminator method) and coherent detection (maximum-likelihood sequence estimation using the Viterbi algorithm, and MSK-type receiver). Coherent detection is employed in GSM base stations and mobile units. In the discussion that follows, perfect carrier- and symbol-timing recovery is assumed.

\section{B. Implementation of a coherent receiver}

The optimum coherent detection for GMSK is by means of the Viterbi algorithm. However, the complexity of the detector grows exponentially with the pulse length $\mathrm{L}$. A sub-optimum receiver with much simpler circuitry is the MSK-type receiver. A MSK-type receiver, which is optimum for MSK, performs reasonably well for binary CPM with $h=1 / 2$. Its performance is almost equal to that of the optimum receiver for schemes with moderate degree of smoothing (e.g., for pulse length up to 3 to 4 symbol intervals) [8]. The GMSK coherent detector in the proposed software radio is based on the principle of MSK-type receiver.

Ignore the effect of the digital matched filter for the moment. The output of the carrier recovery unit in the in-phase arm is $\cos [\phi(k T+\tau, a)]$ where $k T+\tau(\tau$ is the timing offset and $0 \leq \tau \leq T)$ is the sampling instant of the interpolator. For $0.3 \mathrm{GMSK}$, we have $L=4$, so that

$$
\begin{aligned}
\phi(k T+\tau, a) & =\frac{\pi}{2} \sum_{i=-\infty}^{k-4} a_{i}+\pi \sum_{i=k-3}^{k} a_{i} q(k T+\tau-i T) \\
& =\theta(k-2)+I S I(k T+\tau)
\end{aligned}
$$

where

$$
\theta(k)=\frac{\pi}{2} \sum_{i=-\infty}^{k} a_{i}=\frac{\pi}{2} \sum_{i=1}^{k} a_{i}+\theta(0)
$$

$$
\text { and } \begin{aligned}
\operatorname{ISI}(k T+\tau)= & \pi \sum_{i=k-3}^{k-2} a_{i}\left[q(k T+\tau-i T)-\frac{1}{2}\right] \\
& +\pi \sum_{i=k-1}^{k} a_{i} q(k T+\tau-i T) .
\end{aligned}
$$

The phase $\phi(k T+\tau, a)$ consists of an accumulated phase term and an ISI term. The accumulated phase is delibrately delayed by a time $2 \mathrm{~T}$ in order to produce a maximum point at a symmetric pulse of 4T duration. Without loss of generality, let $\theta(0)=0$. The accumulated phase has a phase of either 0 or $\pi$ for $k=2 n$. Therefore, $\theta(2 n)$ can be obtained from $\phi((2 n+2) T+\tau, a)$ by comparing it with a threshold, provided the ISI term can be limited to be less than $\pi / 2$ in the worst case, i.e.

$$
\begin{array}{ll}
|I S I(k T+\tau)|=\left|\begin{array}{l}
\pi \sum_{i=k-3}^{k-2} a_{i}\left[q(k T+\tau-i T)-\frac{1}{2} \mid\right. \\
+\pi \sum_{i=k-1}^{k} a_{i} q(k T+\tau-i T)
\end{array}\right|<\frac{\pi}{2} \\
\Leftrightarrow \quad \sum_{m=0}^{1}|q(m T+\tau)|+\sum_{m=2}^{3}\left|\left[\frac{1}{2}-q(m T+\tau)\right]\right|<\frac{1}{2}
\end{array}
$$

The absolute value of ISI is plotted as a function of $\tau$ in Figure 2. From Figure 2, the ISI term is always smaller than $\pi / 2$. If the output from the interpolator is at the optimum sampling instant, the ISI is a small value. This shows that $\theta(2 n)$ can be recovered by using a simple threshold and a look-up table at time $t=(2 n+2) T+\tau$ in the in-phase arm. A similar situation occurs at the quadrature-phase arm except that it is offset by $\mathrm{T}$ seconds with respect to that of the in-phase arm. That is, $\theta(2 n+1)$ can be obtained from the quadrature-phase arm. As shown in Figure 3 , after $\theta(2 n)$ and $\theta(2 n+1)$ are respectively obtained from the in-phase and quadrature-phase arms, the original data can be recovered by $a_{2 n+1}=(2 / \pi)(\theta(2 n+1)-\theta(2 n))$ or $a_{2 n+2}=(2 / \pi)(\theta(2 n+2)-$ $\theta(2 n+1))$.

The main design challenge is the choice of digital matched filter in order that the original data can be optimally recovered in the in-phase and quadraturephase channels. An obvious choice is to design the filter such that the ISI is removed. From (5), GMSK can be approximated with linear modulation with pulse shape $\mathrm{C}_{0}(\mathrm{t})$. Therefore, $\mathrm{H}_{\mathrm{ISI}-\text {-ree }}(\mathrm{f})=\mathrm{RC}(\mathrm{f}) / \mathrm{C}_{0}(\mathrm{f})$ [10], where $R C(f)$ is the Fourier transform of the raised cosine pulse, is a good candidate for the digital matched filter.

There are various ideas of designing the optimum filter for the analog MSK-type receiver reported in the literature. For example, minimizing the mean- 
square error interference caused by premodulation filtering of the baseband data [9] and minimizing the average error probability [8]. Although optimum filters in analog MSK-type receivers may not be optimum in software radio receivers (since these optimum filters assume carrier recovery being performed before the matched filter while this is not the case in software radios), these criteria may serve as a direction for finding an optimum filter of the software radio.

\section{Implementation of a noncoherent receiver}

GMSK is differentially encoded, so that GMSK signals may be demodulated by noncoherent (differential) methods. Differential detection offers an advantage in a fast fading channel, in which carrier recovery would be difficult to perform. If $\mathrm{H}_{\text {ISI-free }}(\mathrm{f})=\mathrm{RC}(\mathrm{f}) / \mathrm{C}_{0}(\mathrm{f})$ is used as the digital matched filter, the carrier recovery circuit can be replaced by simple differential-detection circuit at the expense of increased sampling rate $(1 / \mathrm{T}$ in both arms). This is illustrated in Figure 4. From (5), at the output of the interpolators, we have

$w_{k}=\cos \left(\frac{\pi}{2} \sum_{n=0}^{k} a_{n}+\alpha\right)$ and $z_{k}=\sin \left(\frac{\pi}{2} \sum_{n=0}^{k} a_{n}+\alpha\right)$

where $\alpha$ is the unknown carrier phase. It follows that

$$
\begin{aligned}
y_{k} & =z_{k} w_{k-1}-w_{k} z_{k-1} \\
& =\sin \left(\frac{\pi}{2} \sum_{n=0}^{k} a_{n}+\alpha\right) \cos \left(\frac{\pi}{2} \sum_{n=0}^{k-1} a_{n}+\alpha\right) \\
& -\cos \left(\frac{\pi}{2} \sum_{n=0}^{k} a_{n}+\alpha\right) \sin \left(\frac{\pi}{2} \sum_{n=0}^{k-1} a_{n}+\alpha\right) \\
& =2 \sin \left(\frac{\pi}{2} a_{k}\right)=\operatorname{sign}\left(a_{k}\right)
\end{aligned}
$$

Therefore, a threshold can be used to recover the data.

\section{CONCLUSIONS}

A practical linear software radio receiver architecture that is suitable for multi-mode operation has been proposed. It has been shown that GMSK can be fitted into the architecture. Coherent and noncoherent detections of GMSK signals are possible for the proposed software radio. This allows the selection of detection method depending on the operating environment, which is one of advantages of the software radio.

\section{ACKNOWLEDGEMENT}

This work was supported by the Hong Kong Research Grants Council and by the University Research Committee of The University of Hong Kong, Hong Kong.

\section{REFERENCES}

[1] J. Mitola, III, "Software radios - survey, critical evaluation and future directions," National Telesystems Conference (NTC92), pp. 13/15 - 13/23, 1992.

[2] M. E. Frerking, Digital Signal Processing in Communication Systems, Van Nostrand Reinhold: New York, 1994.

[3] Intersil Data Sheets: HSP50110, HSP 50210, HSP50214, Intersil Inc.

[4] O. Joeressen, M. Oerder, R. Sërra and H. Meyr, "DIRECS: system design of a 100Mbit/s digital receiver," IEE Proc. pt. $G$, vol.139, pp. 222-230, Apr. 1992.

[5] C.-E. Sundberg, "Continuous phase modulation," IEEE Commun. Mag., pp. 2538, Apr. 1986.

[6] P. Galko and S. Pasupathy, "Linear receiver for correlatively coded MSK," IEEE Trans. Commun., vol.33, pp. 338347, Apr. 1985.

[7] P. A. Laurent, "Exact and approximate construction of digital phase modulations by superposition of amplitude modulated pulses (AMP)," IEEE Trans. Commun., vol. 34, pp. 150-160, Feb. 1986.

[8] A. Svensson and C-E Sundberg, "Optimum MSK-type receivers for CPM on Gaussian and Rayleigh fading channels," IEE Proc. —pt F, vol. 131, pp. 480-490, Aug. 1984.

[9] M. S. El-Tanany and S. A. Mahmoud, "Mean-square error optimization of quadrature receivers for CPM with modulation index 1/2," IEEE J. Select. Areas Commun., vol. 5, pp. 896-905, Jun. 1987.

[10] O. Andrisano and M. Chiani, "The first nyquist criterion applied to coherent receiver design for generalized MSK signals," IEEE Trans. Commun., vol. 42, pp. 449-457, Feb./Mar./Apr. 1994. 


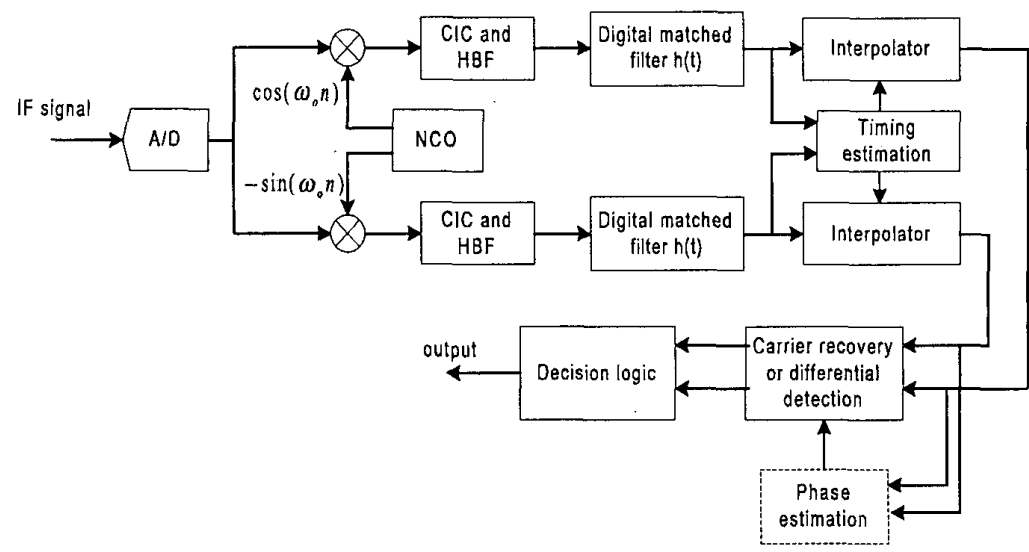

Figure 1. Practical software radio architecture

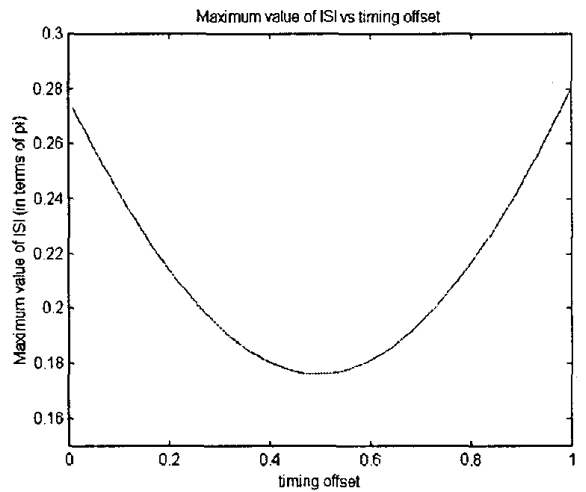

Figure 2. Maximum value of ISI vs timing offset $\tau$

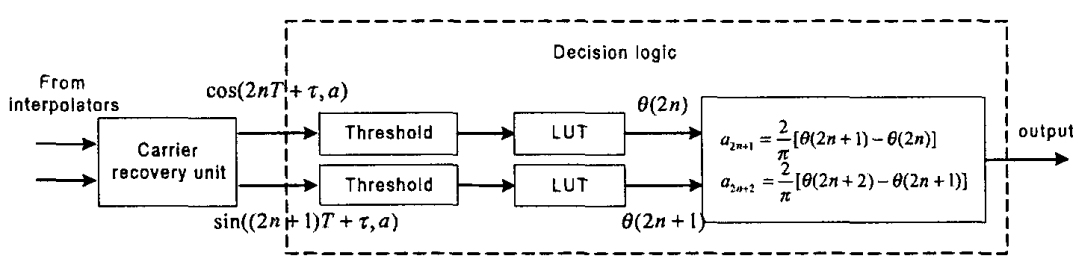

Figure 3. Detail of decision logic for GMSK coherent reception

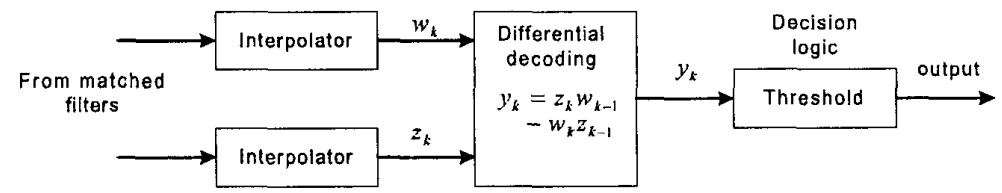

Figure 4. Differential detection for GMSK 